\title{
THE K-BAND WIDE FIELD SURVEY: UNDERSTANDING THE LOCAL GALAXIES
}

\author{
J. HUANG \\ Institute for Astronomy, University of Hawaii
}

\section{The Bright K-band Imaging}

We have conducted a wide-field K-selected galaxy survey with complementary optical I- and B-band imaging in six fields with a total coverage of 9.8 square degrees (Huang et al. 1997). The observations were carried out on the UH $0.6 \mathrm{~m}$ and the UH $2.2 \mathrm{~m}$ telescopes. The purpose of this survey is to study the properties of the local galaxies and explore the evolution of K-selected galaxies at low redshifts. Star-galaxy discrimination is performed using both galaxy color properties and object morphologies, and 6264 galaxies are found. This survey establishes the bright-end K-band galaxy number counts in the magnitude range $13<K<16$ with high precision. We find that our bright-end counts have a significantly steeper slope than the prediction of a no-evolution model, which cannot be accounted for by observational or theoretical error. Since it is very unlikely that there is sufficient evolution at such low redshifts to account for this effect, we argue that there is a local deficiency of galaxies by a factor of 2 on scale sizes of around $300 \mathrm{Mpc}$. This would imply that local measurements of $\Omega_{0}$ underestimate the true value of the cosmological mass density by this factor and that local measurements of $H_{0}$ could be high by as much as $33 \%$.

\section{The Hawaii-Australia K-band Redshift Survey}

We have been granted AAT time to conduct the redshift measurement on our K-selected sample (Huang et al. 1997) by using the 2DF. Actually, one of our fields was observed during the $2 \mathrm{DF}$ commissioning time. There are three primary scientific goals which will be achieved by this redshift survey. 
First, we will construct the local K-band luminosity functions at different redshifts up to $\mathrm{z}=0.2$. By comparing these local luminosity functions, we can determine whether the steep slope of the local K-band counts is caused by a local galaxy deficiency or by galaxy evolution at low redshifts (Huang et al. 1997).

Second, we will be able to determine the evolution of the K-selected galaxies at redshifts $>0.5$. Cowie et al. (1996) derived the K-band luminosity functions at redshifts $>0.5$. The change in the luminosity function can be determined by comparing the K-band luminosity function of Cowie et al. (1996) with the local one.

Finally, we aim to investigate galaxy clustering from a cleanly selected galaxy sample. Previous studies have been based on optical samples (e.g., Loveday et al., 1992) or far-infrared IRAS galaxies (e.g., Dekel et al., 1993), both of which are inherently biased to star-forming systems and are already known to avoid rich clusters. This has led to much dispute about $b$, the biasing parameter of light relative to mass. A K-selected sample is inherently superior in that the $\mathrm{K}$ band light is a close tracer of underlying stellar mass rather than star-formation. This has long been recognized as desirable and has led to large all-sky K surveys such as 2MASS and DENIS; these surveys, however, are part of longer term efforts. My large contiguous areas will allow investigation of galaxy clustering on scale $\leq 20 \mathrm{~h}^{-1}$ Mpc. Although the volume surveyed is smaller then some of the $>10^{5}$ optically selected redshift surveys, it will provide a valuable check on their results and allow their biases as a function of morphology and color to be assessed. Uniquely, this wide field K-band survey will also allow us to investigate the evolution of clustering to $\mathrm{z}=0.2$. Not only do we have an unchanging morphological mix with redshift, we also see the same population as at $\mathrm{z}=0$. Again, this contrasts with optically selected surveys-at $\mathrm{z}=0.2$ they are dominated by small starburst systems, a different population whose clustering properties may well be intrinsically different (Efstathiou et al. 1991).

\section{The Morphology Classification and Surface Photometry of the $\mathrm{K}$-selected galaxies}

The morphological mix of the K-selected galaxy sample has yet to be determined. Huang et al. (1997) obtained the morphological mix from the mean color-magnitude relation. By fitting the mean color-magnitude relation, they conclude that a K-selected galaxy sample contains mainly elliptical and early type spiral galaxies, but very few irregular galaxies. This conclusion needs to be confirmed by the morphological classification. 
The morphology mix is a key factor in understanding the galaxy evolution and modeling the galaxy number counts. With the morphological classification and redshifts measured in the $2 \mathrm{DF}$ redshift survey, we will be also able to construct the local type-dependent luminosity functions. These type-dependent luminosity functions will allows us to model the number counts more precisely to understand galaxy evolution.

Large format CCDs have provided an effective way for us to obtain high $\mathrm{S} / \mathrm{N}$ images for a sample with wide coverage. An $8 \mathrm{k} \times 8 \mathrm{k}$ CCD has been built by UH astronomers, and is now available on the CFHT and the UH 88 -inch telescopes. The field of view is about $0.5^{\circ}$ with a scale of $0.22^{\prime \prime}$ pixel $^{-1}$. One of our fields has been observed in R-band on the CFHT with the $8 \mathrm{k} \times 8 \mathrm{k}$ CCD. The exposure time is one hour, and the seeing is 0.7 . The image can allow us to clearly classify the morphologies of our K-selected sample to $K=15$.

Surface photometry of the K-selected sample is another goal of the $8 \mathrm{k}$ CCD imaging. Recently surface photometry (Schade et al. 1996) of optically selected samples has led some authurs to conclude there is luminosity evolution of the spiral galaxies. However, the redshift distributions from other redshift surveys do not support this conclusion. We will be able to reinvestigate this issue by using the $\mathrm{K}$-selected sample. Our advantages are the high $\mathrm{S} / \mathrm{N}$, larger sample and insensitivity of the K-selected sample to star-formation.

\section{The QUick Infrared Galaxy Survey}

The K-band galaxy number counts below $\mathrm{K}=13$ remain controversial. Two current K-band surveys (Huang et al. 1997, Gardner et al. 1996) have too limited sky coverage (both cover about 10 square degrees) to obtain precise galaxy number counts below $\mathrm{K}=13$. Though Mobasher et al. (1986) conducted a substantial K-band survey below $K=12$, their sample was selected from the B-selected sample and might suffer from some systematic errors. A K-band survey with a sky coverage of 300 square degrees is required to solve this problem.

Though there will be large near-infrared surveys such as the 2MASS and DENIS, The QUick Infrared Galaxy survey is designed to attack this problem easily and effectively. The University of Hawaii has built the QUick Infrared Survey Telescope (QUIST). QUIST is a $25-\mathrm{cm} \mathrm{f} / 10$ infrared optimized telescope which mounts on top of QUIRC (QUick InfraRed Camera). QUIRC has a $1024 \times 1024 \mathrm{HgCdTe}$ infrared array sensitive from 0.7 to 2.5 microns. QUIST with QUIRC are mounted in binocular fashion with the University of Hawaii's 61-cm telescope located on Mauna Kea. QUIST has a field of view of $0.43 \times 0.43$ degrees and pixels are 1.5 arcseconds across. 
The observation on the QUIST is totally remote-controlled. Please check the IfA web page for more detailed information and the QUIST images (http://www.ifa.hawaii.edu/images/quist/).

This survey has been in operation since May, 1996. We find that a total exposure of 6 minutes can allow us to detect a galaxy with $\mathrm{K}=14$. So far about 30 square degrees of sky has been observed. We expect to finish this survey before the end of this year.

\section{References}

Cowie, L. L., Songaila, A., \& Hu, E. M. 1996, Astron.J., In press

Dekel, A., et al. 1993, Astrophys.J. 412, 1

Efstathious, G., et al. 1991, Astrophys.J. 380, L47

Gardner, J. P., et al. 1996, Mon.Not.R.astron.Soc., in press.

Huang, J-S., et al. 1997, in press

Loveday, J., et al. 1992, Mon.Not.R.astron.Soc., 247, 1P

Mobasher, B., Ellis, R. S., \& Sharples, R. M. 1986, Mon.Not.R.astron.Soc., 223, 11 